\title{
The efficacy and safety of Carbon ion radiotherapy for Meningiomas: A Systematic review and meta-analysis
}

\author{
Li Jieyun ${ }^{1}$, Jingwen $\mathrm{Li}^{1}$, meixuan $\mathrm{Li}^{1}$, yuanchang $\mathrm{Jin}^{1}$, Qiuning Zhang ${ }^{1}$, Fei Bai ${ }^{1}$, Xiuxia \\ $\mathrm{Li}^{1}$, Xiaohu Wang ${ }^{1}$, and Kehu Yang ${ }^{2}$
}

${ }^{1}$ Affiliation not available

${ }^{2}$ Evidence Based Medicine Center

November 7, 2020

\begin{abstract}
Background: Traditional radiotherapy is difficult to carry out definite treatment without damaging the adjacent brain parenchyma. Many studies have shown that the physical properties of carbocation radiation therapy allow treatment to reduce damage to critical structures and thus improve patient survival. Purpose: The purpose of this systematic review and meta-analysis is to evaluate the efficacy and safety of Carbon ion or Carbon ion combination radiation therapy in improving meningioma. Materials and Methods: The related studies published from January 1, 1951 to August 1, 2020 were searched comprehensively on PubMed, Cochrane Library, China Biomedical Literature Database, Web of Science, EMBASE. The extracted data included studies focused on rates and types of adverse events were sorted and classified by excel, and the overall survival and local control rates data were further analyzed with R software, Results: In eight included studies and 506 individuals, the three-year survival rate and annual local control rate of benign meningiomas were 100\%. The one-year, two-year and 5-year survival rates of patients with atypical meningioma were $100 \%, 95 \%$ and $50 \%$. The one-year and two-year survival rates of patients with recurrent intracranial meningiomas were $90 \%$ and $71 \%$ respectively. The one-year and two-year local control rates of non-benign meningiomas were $53 \%$ and $33 \%$ respectively. Headache, sensory impairment, cognitive impairment, and hearing impairment were the most common toxic reactions, with incidences of $19.4 \%, 23.7 \%, 9.1 \%$ and $9.1 \%$, respectively.
\end{abstract}

\section{Hosted file}

manuscript.pdf available at https://authorea.com/users/344317/articles/491524-the-efficacyand-safety-of-carbon-ion-radiotherapy-for-meningiomas-a-systematic-review-and-metaanalysis 


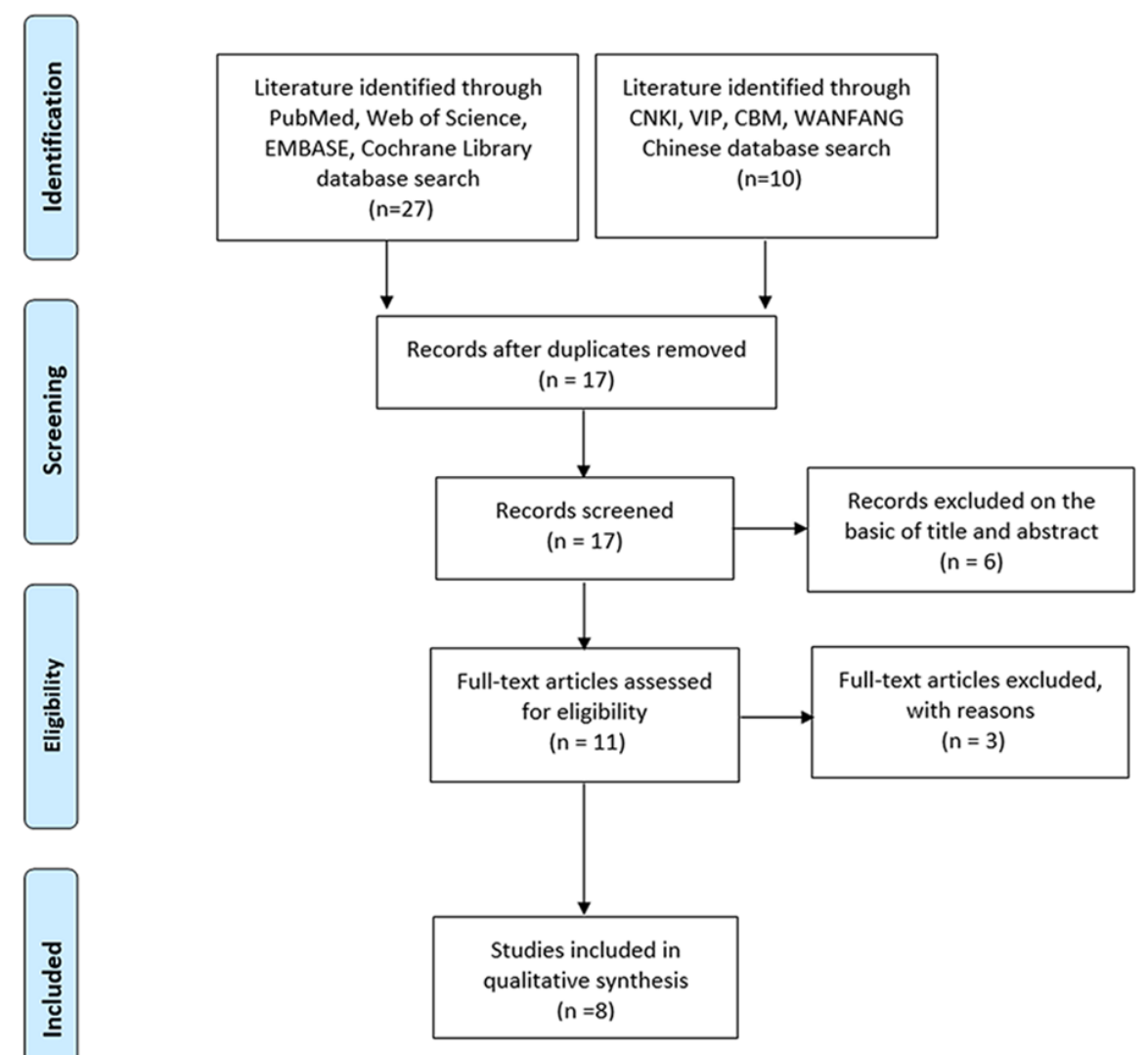




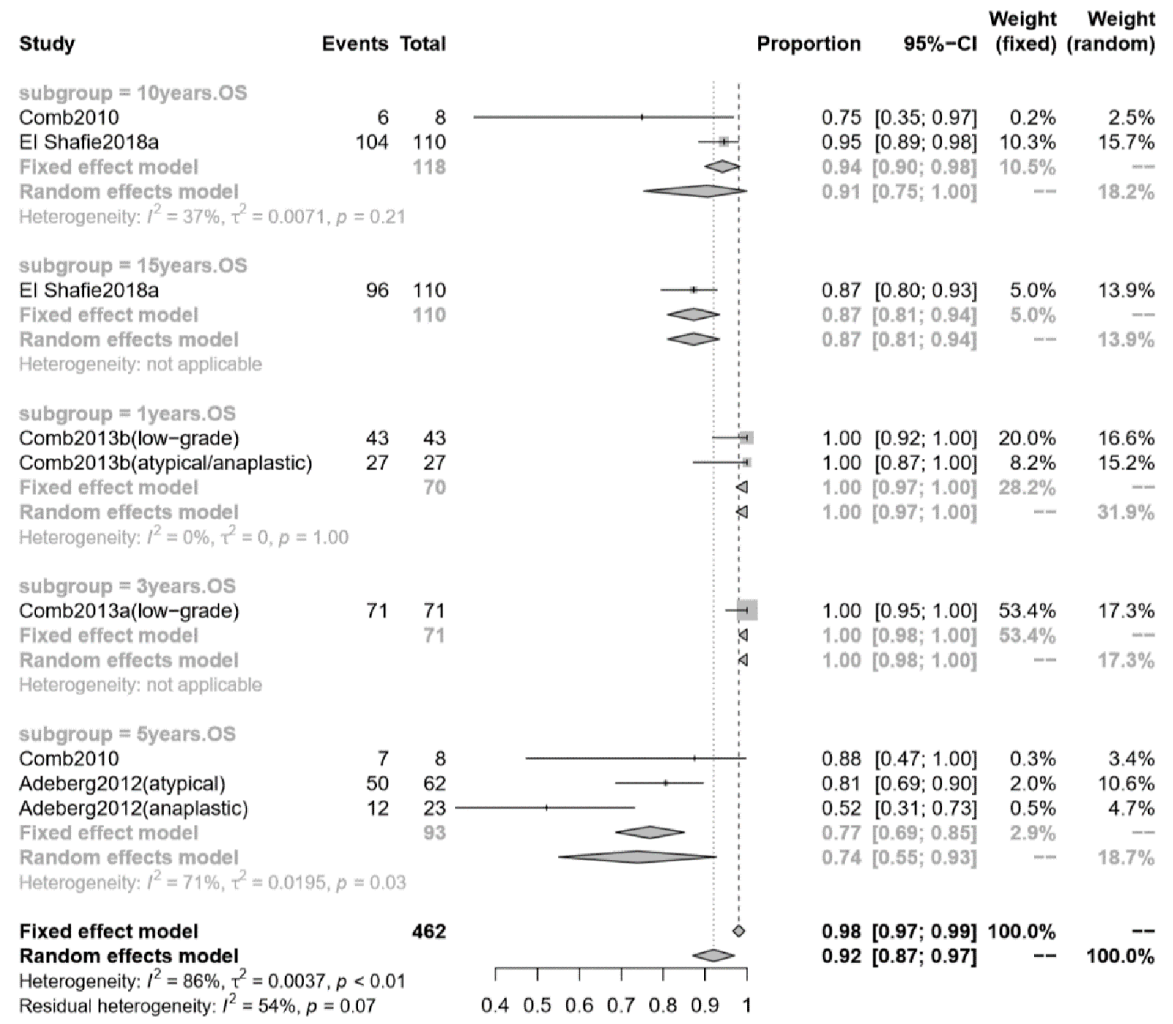

\section{Study}

subgroup $=1$ years, $\mathrm{LC}$ Comb2013a(atypicalanaplastic) Fixed effect model

Random effects model

Heterogeneity: not applicable

subgroup = 2years.LC Comb2013a(atypicalanaplastic)

Fixed effect model

Random effects model

Heterogeneity: not applicable

subgroup $=3$ years. LC

Comb2013a(low-grade)

Fixed effect model

Random effects model

Heterogeneity: not applicable

subgroup $=5$ years. LC

Comb2010

Fixed effect model

Random effects model

Heterogeneity: not applicable

subgroup $=7$ years. $\mathrm{LC}$

Comb2010

Fixed effect model

Random effects model

Heterogeneity: not applicable

Fixed effect mode

Random effects model

Heterogeneity: $I^{2}=96 \%, \tau^{2}=0.1245, p<0.01$
Residual heterogeneity. $I^{2}=\mathrm{NA} \%, p=\mathrm{NA}$

12

(n)

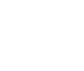

6

\section{Events Total}

$19 \quad 36$

36
36

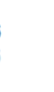
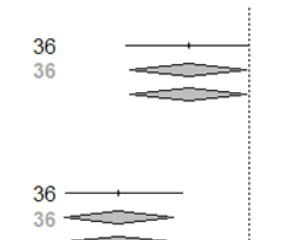

36

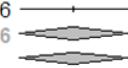

$\begin{array}{ll}71 & 71\end{array}$

1
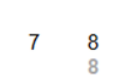

8

8
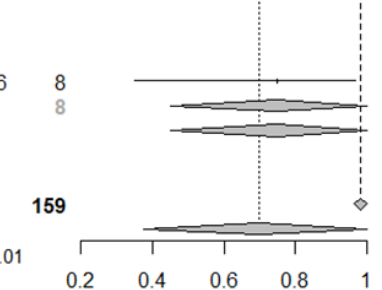

Weight Weight

Proportion $\quad 95 \%-\mathrm{Cl} \quad$ (fixed) (random)

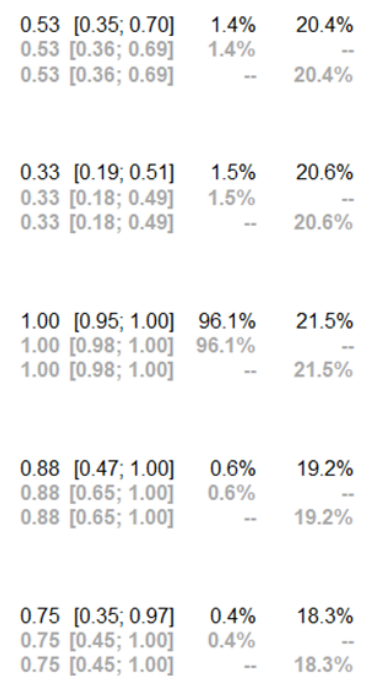

$0.53[0.35 ; 0.70] \quad 1.4 \% \quad 20.4 \%$ $0.53[0.36 ; 0.69] \quad 1.4 \%$

$0.53[0.36 ; 0.69] \quad--\quad 20.4 \%$

$0.33[0.19 ; 0.51] \quad 1.5 \% \quad 20.6 \%$

$0.33[0.18 ; 0.49] \quad 1.5 \% \quad 20.6 \%$

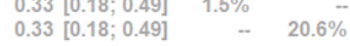

$1.00[0.95 ; 1.00] \quad 96.1 \% \quad 21.5 \%$

$\begin{array}{rrr}1.00[0.98 ; 1.00] & 96.1 \% & - \\ 1.00[0.98 ; 1.00] & - & 21.5 \%\end{array}$

$0.88[0.47 ; 1.00] \quad 0.6 \% \quad 19.2 \%$

$0.88[0.65 ; 1.00] \quad 0.6 \% \quad-$

$0.88[0.65 ; 1.00] \quad-\quad 19.2 \%$

$0.75[0.35 ; 0.97] \quad 0.4 \% \quad 18.3 \%$

$\begin{array}{lrr}0.75[0.45 ; 1.00] & 0.4 \% & - \\ 0.75[0.45 ; 1.00] & -- & 18.3 \%\end{array}$

$0.98[0.96 ; 1.00] 100.0 \%$

$0.70[0.38 ; 1.00] \quad-\quad 100.0 \%$ 